\title{
Universality classes in Burgers turbulence
}

\author{
Govind Menon ${ }^{1}$ and Robert. L. Pego ${ }^{2}$
}

September 9, 2017

\begin{abstract}
We establish necessary and sufficient conditions for the shock statistics to approach self-similar form in Burgers turbulence with Lévy process initial data. The proof relies upon an elegant closure theorem of Bertoin and Carraro and Duchon that reduces the study of shock statistics to Smoluchowski's coagulation equation with additive kernel, and upon our previous characterization of the domains of attraction of self-similar solutions for this equation.
\end{abstract}

Keywords: Burgers turbulence, Smoluchowski's coagulation equation, Lévy processes, dynamic scaling, regular variation, agglomeration, coagulation, coalescence, shock statistics.

MSC classification: 60J75, 35R60, 35L67, 82C99

\footnotetext{
${ }^{1}$ Division of Applied Mathematics, Box F, Brown University, Providence, RI 02912. Email: menon@dam.brown.edu

${ }^{2}$ Center for Nonlinear Analysis, Department of Mathematical Sciences, Carnegie Mellon University, Pittsburgh, PA 15213. Email: rpego@cmu.edu
} 


\section{Introduction}

The construction of stochastic processes that are also weak solutions to the equations of fluid mechanics is one approach to rigorous mathematical theories of turbulence. This is poorly understood at present, and we must settle for insights from vastly simplified model problems. We consider the invisicid Burgers equation

$$
\partial_{t} u+\partial_{x}\left(\frac{u^{2}}{2}\right)=0, \quad t>0, x \in \mathbb{R}, \quad u(x, 0)=u_{0}(x),
$$

with random initial data $u_{0}$. The problem is to determine the statistical properties of the Cole-Hopf (entropy) solution $u(x, t)$ to $(1)$, given the statistical properties of $u_{0}$. There is a large literature on the subject; we refer to Burgers' book [10] and the more recent survey articles [17, 25, 36]. The problem was proposed by Burgers as a model for turbulence in incompressible fluids, but it has several well-known flaws in this regard.

Explicit solutions play a special role in the theory. Burgers studied the case when $u_{0}$ is white noise in his monograph [10]. His work remains the foundation for several rigorous results, which culminate with the complete solution by Frachebourg and Martin for the velocity and shock statistics (see [19] and references therein). The case when $u_{0}$ is a Brownian motion has attracted much attention since the work of She, Aurell and Frisch [34] and Sinai [35]. An elegant solution to this problem was obtained by Bertoin [5] and Carraro and Duchon [12]. More generally, these authors considered initial data that comprise a Lévy process with only downward jumps (i.e., shocks). A Lévy process $X_{x}(x \geq 0)$ is a continuous-time random walk with stationary and independent increments. It is determined completely by its characteristic exponent $\Psi$, satisfying $\mathbb{E}\left(e^{i k X_{x}}\right)=e^{-x \Psi(k)}$, via the celebrated Lévy-Khintchine formula

$$
\Psi(k)=i b k+\frac{\sigma^{2} k^{2}}{2}+\int_{\mathbb{R}}\left(1-e^{i k s}+i k s \mathbf{1}_{|s|<1}\right) \Pi(d s), \quad k \in \mathbb{R} .
$$

The process $X$ is the superposition of three independent processes related to this formula: a Brownian motion with variance $\sigma^{2}$ and drift $b \in \mathbb{R}$, a compound Poisson process with jump measure $\Pi \mathbf{1}_{|s| \geq 1}$, and a pure jump martingale with jump measure $\Pi \mathbf{1}_{|s|<1}$ (see [4, Ch.1]). The measure $\Pi$ is arbitrary, subject to the condition $\int_{\mathbb{R}}\left(1 \wedge s^{2}\right) \Pi(d s)<\infty$, where $a \wedge b$ means $\min (a, b)$. 
We say $X$ is spectrally negative if $\Pi$ is concentrated on the half-line $s<0$. In all that follows we assume

$$
u_{0}(x)=\left\{\begin{array}{l}
0, \quad x<0, \\
\text { a spectrally negative Lévy process, } \quad x \geq 0
\end{array}\right.
$$

As we show below, we can always reduce to the case where $u_{0}(x)$ has zero mean $\mathbb{E}\left(u_{0}(x)\right)$ for all $x$, and $\int_{-\infty}^{0}\left(|s| \wedge s^{2}\right) \Pi(d s)<\infty$. It is then more convenient to use the Laplace exponent

$$
\psi(q)=-\Psi(-i q)=\frac{\sigma^{2} q^{2}}{2}+\int_{0}^{\infty}\left(e^{-q s}-1+q s\right) \Lambda(d s), \quad q>0,
$$

where $\Lambda((s, \infty))=\Pi((-\infty,-s))$ for every $s>0$, so that $\mathbb{E}\left(e^{q X_{x}}\right)=e^{x \psi(q)}$ and $\mathbb{E}\left(X_{x}\right)=x \psi^{\prime}(0)=0$.

For this class of initial data, Bertoin proved a remarkable closure property for the entropy solution of (1), namely: $x \mapsto u(x, t)-u(0, t)$ remains a spectrally negative Lévy process for all $t>0$. This closure property was first noted by Carraro and Duchon in connection with their notion of statistical solutions to Burgers equation [11]. That these statistical solutions agree with the Cole-Hopf solution for spectrally negative data was shown by Bertoin [ 5 , Thm. 2]. The closure property fails if $u_{0}$ has positive jumps-These positive jumps open into rarefaction waves for $t>0$, and this is incompatible with the rigidity of sample paths of Lévy processes. An interesting formal analysis of closure properties of Burgers equation is presented in [13].

Henceforth, we write $v(x, t)=u(x, t)-u(0, t)$ for brevity. The LévyKhintchine representation now implies that the law of the Lévy process $x \mapsto$ $v(x, t)$ is completely described by a corresponding "Lévy triplet" $\left(b_{t}, \sigma_{t}^{2}, \Lambda_{t}\right)$. The mean drift $b_{t}=\mathbb{E}(v(1, t))$ satisfies $b_{t}=b_{0}=0$ for every $t \geq 0$. Moreover, for every $t>0, v(\cdot, t)$ is of bounded variation, thus the variance $\sigma_{t}=0$. Consequently, the law of $v(\cdot, t)$ is completely determined by only the jump measure $\Lambda_{t}$ which contains the shock statistics.

It is a striking fact, implicit in [5], that the evolution of $\Lambda_{t}$ is described by Smoluchowski's coagulation equation with additive kernel, an equation that arises in entirely different areas such as the analysis of algorithms [14], the kinetics of polymerization [37], and cloud formation from droplets [23] (see [2] for a review). What this means is that mean-field theory is exact for Burgers equation with initial data of the form (3), i.e., random onesided data with stationary and independent increments. We give a precise statement to this effect below in Theorem 2. Several connections between stochastic models of coalescence and Burgers turbulence are reviewed in [8]. 
Here, we use the closure property as a basis for a rigorous study of universality classes for dynamic scaling in Burgers turbulence. Our motivation is the following. A central theme in studies of homogeneous isotropic turbulence in incompressible fluids is the universality of the Kolmogorov spectrum [30]. A possible rigorous formulation of such universality involves (a) the construction of stochastic processes that mimic a 'typical turbulent flow', and (b) a characterization of the domains of attraction of these processes. For Burgers turbulence, step (a) consists of constructing exact solutions for special initial data, say white noise or Brownian motion. In this article, we carry out step (b) for initial data that satisfy (3).

Domains of attraction are studied in the classical limit theorems in probability (e.g., the central limit theorem), and their process versions (e.g., Donsker's invariance principle). For Smoluchowski's coagulation equation with additive kernel, we characterized all possible domains of attraction in [32], a result akin to the classical limit theorems. In this article we deal with a process version. In all that follows, we consider the processes $x \mapsto v(x, t)$ as elements of the space $\mathbb{D}$ of right continuous paths $\mathbb{R}_{+} \rightarrow \mathbb{R}$ with left limits (càdlàg paths) equipped with the Skorokhod topology [28, Ch. VI]. The shock statistics determine completely the law of this process (a probability measure on $\mathbb{D}$ ). Approach to limiting forms will be phrased in terms of weak convergence of probability measures on $\mathbb{D}$.

Among the initial data we consider, the stable processes are of particular importance because of their self-similarity. Let $X^{\alpha}, \alpha \in(1,2]$ denote the stable process with Laplace exponent $q^{\alpha}$ ( $\alpha=2$ corresponds to Brownian motion). The corresponding jump measure $\Lambda(d s)=s^{-1-\alpha} d s / \Gamma(-\alpha)$ for $\alpha<$ 2. There is a one-to-one correspondence between (a) these stable processes, (b) statistically self-similar solutions in Burgers turbulence, and (c) selfsimilar solutions to Smoluchowski's coagulation equation. Precisely, this works as follows. Let $\alpha \in(1,2]$, and let $T^{\alpha}$ denote the first-passage process for $x \mapsto X_{x}^{\alpha}+x$, i.e.,

$$
T_{x}^{\alpha}=\inf \left\{y \mid X_{y}^{\alpha}+y>x\right\} .
$$

Then for the solution to (1) with $u_{0}(x)=X_{x}^{\alpha}$ for $x \geq 0, v(x, t)$ is statistically self-similar, with

$$
v(x, t) \stackrel{\mathcal{L}}{=} t^{1 / \beta-1} V_{x t^{-1 / \beta}}^{\alpha}:=t^{1 / \beta-1}\left(x t^{-1 / \beta}-T_{x t^{-1 / \beta}}^{\alpha}\right), \quad t, x>0,
$$

where $\beta=(\alpha-1) / \alpha$. Here $\stackrel{\mathcal{L}}{=}$ means both processes define the same measure on $\mathbb{D}$. The process $T^{\alpha}$ is a pure jump Lévy process with Lévy measure 
$f_{\alpha}(s) d s$, where $f_{\alpha}$ is the number density profile of a self-similar solution to Smoluchowski's coagulation equation [32, Sec 6]:

$$
f_{\alpha}(s)=\frac{1}{\pi} \sum_{k=1}^{\infty} \frac{(-1)^{k-1} s^{k \beta-2}}{k !} \Gamma(1+k-k \beta) \sin \pi k \beta, \quad \alpha \in(1,2] .
$$

These solutions are related to classical distributions in probability theory by rescaling. If $p(s ; \alpha, 2-\alpha)$ denotes the density of a maximally-skewed Lévy stable law [18, XVII.7] we have [7, 32]

$$
f_{\alpha}(s)=s^{\beta-2} p\left(s^{\beta} ; \alpha, 2-\alpha\right) .
$$

By the Lévy-Itô decomposition [4, Thm 1.1] and (6) we may conclude that the magnitudes of shocks in $u(\cdot, t)$ form a Poisson point process valued in $(0, \infty)$ whose characteristic measure is

$$
\Lambda_{t}^{\alpha}(d s)=t^{1-2 / \beta} f_{\alpha}\left(s t^{1-1 / \beta}\right) d s .
$$

For $1<\alpha<2$ the self-similar solutions have algebraic tails, with $f_{\alpha}(s) \sim$ $s^{-1-\alpha} / \Gamma(-\alpha)$ as $s \rightarrow \infty$. The case $\alpha=2$ is particularly important since it corresponds to Brownian initial data. Here we obtain a solution found by Golovin in a model for cloud formation from droplets [23],

$$
f_{2}(s)=(4 \pi)^{-1 / 2} s^{-3 / 2} e^{-s / 4} .
$$

For the corresponding solution to (1), the law of $v(x, t)$ can be recovered from the law of $T_{x}^{2}$, the first-passage time for Brownian motion with unit drift, which is explicitly given as follows (see section 2.6):

$$
\mathbb{P}\left(T_{x}^{2} \in(y, y+d y)\right)=\frac{x \mathbf{1}_{y>0}}{2 \sqrt{\pi y^{3}}} \exp \left(-\frac{(x-y)^{2}}{4 y}\right) d y .
$$

Considering now arbitrary solutions to (1) with initial data (3), we classify solutions that approach self-similar form as $t \rightarrow \infty$ as follows. We establish necessary and sufficient conditions for convergence of the laws of the rescaled processes

$$
x \mapsto V_{x}^{(t)}=\frac{t}{\lambda(t)} v(\lambda(t) x, t)
$$

in the sense of weak convergence of measures on $\mathbb{D}$. (Since the shocks coalesce, a rescaling $\lambda(t) \rightarrow \infty$ is needed to obtain a non-trivial limit. The amplitude scaling is natural, see Section 3.) Convergence to a process $V^{*}$ is written $V^{(t)} \stackrel{\mathcal{L}}{\rightarrow} V^{*}$ as in [28]. Recall that a positive function $L$ is said to be slowly varying at $\infty$ if $\lim _{t \rightarrow \infty} L(t x) / L(t)=1$ for all $x>0$. 
Theorem 1. Let $u_{0}$ be a spectrally negative Lévy process with zero mean $\mathbb{E}\left(u_{0}(x)\right)$, variance $\sigma_{0}^{2} \geq 0$, and downward jump measure satisfying $\int_{0}^{\infty}(s \wedge$ $\left.s^{2}\right) \Lambda_{0}(d s)<\infty$.

1. Suppose there is a rescaling $\lambda(t) \rightarrow \infty$ as $t \rightarrow \infty$ and a Lévy process $V^{*}$ with zero mean $\mathbb{E}\left(V_{1}^{*}\right)$ such that the random variables $V_{1}^{(t)}$ converge to $V_{1}^{*}$ in law. Then there exists $\alpha \in(1,2]$ and a function $L$ slowly varying at infinity such that

$$
\sigma_{0}^{2}+\int_{0}^{s} r^{2} \Lambda_{0}(d r) \sim s^{2-\alpha} L(s) \quad \text { as } s \rightarrow \infty .
$$

2. Conversely, assume that there exists $\alpha \in(1,2]$ and a function $L$ slowly varying at infinity such that (13) holds. Then there is a strictly increasing rescaling $\lambda(t) \rightarrow \infty$ such that $V^{(t)} \stackrel{\mathcal{L}}{\rightarrow} V^{\alpha}$.

Remark 1. Since $V^{(t)}$ and $V^{*}$ are Lévy processes, we have $V^{(t)} \stackrel{\mathcal{L}}{\rightarrow} V^{*}$ if and only if we have convergence in law of the random variables $V_{x_{0}}^{(t)}$ for some fixed $x_{0} \in(0, \infty)$ (see (58)-(59) in section 3 below). We take $x_{0}=1$ without loss of generality. Part (2) implies in particular that the only possible limits are statistically self-similar.

Remark 2. We say a solution has finite energy if for any finite interval $I \subset \mathbb{R}_{+}$we have $\mathbb{E}\left(\int_{I}|v(x, t)|^{2} d x\right)<\infty$. The jump measure $\Lambda_{t}$ for the solution is related to the energy by (see Section 4 )

$$
\mathbb{E}\left(\int_{I} v(x, t)^{2} d x\right)=\left(\int_{0}^{\infty} s^{2} \Lambda_{t}(d s)\right) \int_{I} x d x
$$

The integral in (13) is thus a measure of the energy in an interval. If it is initially finite, it is conserved for $t>0$, and it remains infinite if it is initially infinite. The only self-similar solution with finite energy corresponds to $\alpha=2$, and Theorem 1 implies it attracts all solutions with initially finite energy. In this sense, one may say that the finite energy solution is universal. However, Theorem 1 also indicates the delicate dependence of the domains of attraction on the tail behavior of $\Lambda_{0}$. Heavy-tailed solutions seem to us no less interesting than those with finite energy. Finer results on asymptotics, and a compactness theorem for subsequential limits that builds on Bertoin's Lévy-Khintchine classification for eternal solutions to Smoluchowski's equation [7], will be developed elsewhere. 
Remark 3. The case of zero mean, $b_{0}=0$, is the most interesting. If $b_{0}>0$ or $b_{0}<0$ we can reduce to this case by a change of variables (see below). If $b_{0}<0$, the solution is defined only for $0 \leq t<-b_{0}^{-1}$. Theorem 1 then characterizes the approach to self-similarity at the blow-up time. If $b_{0}>0$ then the behavior of the solution as $t \rightarrow \infty$ is determined by the zero-mean solution with the same $\sigma_{0}^{2}$ and $\Lambda_{0}$ at the finite time $b_{0}^{-1}$.

Remark 4. The Cole-Hopf solution is geometric and Theorem 1 may be a viewed as a limit theorem for statistics of minima. The utility of regular variation in such problems is widely known [33]. If the initial data is white noise, the Cole-Hopf solution is a study of the parabolic hull of Brownian motion. Groeneboom's work on this problem [24] is the basis for several results on Burgers turbulence (in particular [3, 19, 22]). We have been unable to find a similar reference to the problem we consider in the probability literature ([9] seems the closest).

Remark 5. There is a growing literature on intermittence, and the asymptotic self-similarity of Burgers turbulence, see for example [21, 26]. Numerical simulations and heuristic arguments suggest that this is a subtle problem with several distinct regimes. It is hard to obtain rigorous results for general initial data. Theorem 1 tells us that the approach to self-similarity is at least as complex as in the classical limit theorems of probability.

The rest of this article is organized as follows. We explain the mapping from Burgers equation to Smoluchowski's coagulation equation in Section 2. This is followed by the proof of Theorem 1 in Section 3. Finally, in Section 4 we compute a number of statistics of physical interest: energy and dissipation in solutions, the Fourier-Laplace spectrum, and the multifractal spectrum.

\section{Mean field theory for Burgers equation}

In this section we explain the connection between Burgers equation with spectrally negative Lévy process data and Smoluchowski's coagulation equation. The main results are due to Bertoin [5] and Carraro and Duchon [12]. We follow Bertoin's approach, and explain results implicit in [5] and [7]. We think it worthwhile to make this connection widely known in full generality, since the results are of interest to many non-probabilists. Exact solutions of this simplicity are also useful as benchmark problems for numerical calculations. 


\subsection{Shock coalescence and Smoluchowski's coagulation equa- tion}

Smoluchowski's coagulation equation is a widely used mean-field model of cluster growth (see $[2,16]$ for introductions). We begin with a heuristic derivation of the coagulation equation as a mean-field model of shock coalescence. First consider the evolution of a single shock of size $s>0$. Let $u_{0}(x)=-s \mathbf{1}_{x \geq 0}$. Then the solution is

$$
u(x, t)=-s \mathbf{1}_{x \geq x_{1}(t)}, \quad x_{1}(t)=-\frac{s}{2} t .
$$

Shock coalescence is nicely seen as follows. Let $u_{0}(x)=-\sum_{k=1}^{N} s_{k}(0) \mathbf{1}_{x \geq x_{k}(0)}$ where $s_{k}(0)>0$ for $k=1, \ldots, N$ and $x_{1}(0)<\ldots x_{N}(0)$. The solution may be constructed using the method of characteristics and the standard jump condition

$$
\dot{x}=\frac{1}{2}\left(u^{-}+u^{+}\right)
$$

across a shock at $x=x(t)$, where $u^{-}$and $u^{+}$denote respectively the left and right limits of $u(\cdot, t)$ at $x$. At any time $t>0$, there are $N(t) \leq N(0)$ shocks at locations $x_{1}(t)<x_{k}(t)<x_{N(t)}(t)$ and

$$
u(x, t)=-\sum_{k=1}^{N} s_{k}(t) \mathbf{1}_{x \geq x_{k}(t)}, \quad \dot{x}_{k}\left(t_{+}\right)=-\sum_{j=1}^{k-1} s_{j}\left(t_{+}\right)-\frac{s_{k}\left(t_{+}\right)}{2} .
$$

The shock sizes $s_{k}(t)$ are constant between collisions, and add upon collisionwhen shocks $k$ and $k+1$ collide, we set $s_{k}\left(t_{+}\right)=s_{k}\left(t_{-}\right)+s_{k+1}\left(t_{-}\right)$and relabel. This yields an appealing sticky particle or ballistic aggregation scenario. We say a system of particles with position, mass and velocity $\left(x_{k}(t), m_{k}(t), v_{k}(t)\right)$ undergoes ballistic aggregation if (a) the particles move with constant mass and velocity between collisions, and (b) at collisions, the colliding particles stick to form a single particle, conserving mass and momentum in the process. We map this shock coalescence problem to a sticky particle system by setting $m_{k}=s_{k}$ and $v_{k}=\dot{x}_{k}$. Suppose particles $k$ and $k+1$ meet at time $t$. Then, with unprimed variables denoting values before collision and primed variables denoting values after, since $v_{k+1}=v_{k}-\left(m_{k}+m_{k+1}\right) / 2$ we have

$$
m_{k} v_{k}+m_{k+1} v_{k+1}=\left(m_{k}+m_{k+1}\right)\left(v_{k}-\frac{m_{k+1}}{2}\right)=m_{k}^{\prime} v_{k}^{\prime} .
$$

The calculations so far involve no randomness. Suppose now that the shock sizes $s_{j}$ are independent and let $f(s, t) d s$ denote the expected number 


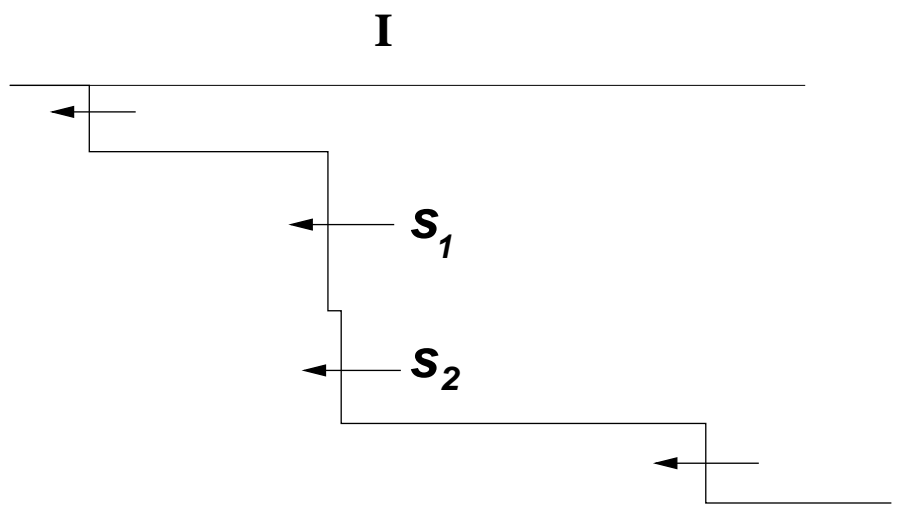

Figure 1: Binary clustering of shocks

of shocks per unit length with size in $[s, s+d s]$. We derive a mean-field rate equation for $f$ as follows. Let $I$ be an interval of unit length. The number density changes because of the flux of shocks entering and leaving $I$ and because of shock collisions within $I$. On average, the velocity difference across $I$ is

$$
M_{1}(t)=\int_{0}^{\infty} s f(s, t) d s,
$$

therefore the average influx is $M_{1}(t) f(s, t) d s$. Next consider the formation of a shock of size $s_{1}+s_{2}$ by a collision of shocks of size $s_{1}$ and $s_{2}$ as shown in Figure 1. The relative velocity between these shocks is $\left(s_{1}+s_{2}\right) / 2$ (see Figure 1). The expected number of neighboring pairs with sizes in $\left[s_{1}, s_{1}+\right.$ $\left.d s_{1}\right],\left[s_{2}, s_{2}+d s_{2}\right]$ respectively is

$$
f\left(s_{1}, t\right) f\left(s_{2}, t\right) d s_{1} d s_{2} .
$$

The probability that these neighboring shocks are near enough to collide in time $d t$ is $\frac{1}{2}\left(s_{1}+s_{2}\right) d t$, thus the number of these shocks that collide in time $d t$ is

$$
f\left(s_{1}, t\right) f\left(s_{2}, t\right) \frac{s_{1}+s_{2}}{2} d s_{1} d s_{2} d t .
$$

Summing over all collisions that create shocks of size $s=s_{1}+s_{2}$, and accounting for the loss of shocks of size $s\left(=s_{1}\right.$ or $\left.s_{2}\right)$ in collisions with other shocks, we obtain the rate equation

$$
\partial_{t} f(s, t)=M_{1}(t) f+Q(f, f)
$$

where $Q(f, f)$ denotes the collision operator given by

$Q(f, f)(s, t)=\frac{1}{2} \int_{0}^{s} s f\left(s_{1}, t\right) f\left(s-s_{1}, t\right) d s_{1}-\int_{0}^{\infty}\left(s+s_{1}\right) f(s, t) f\left(s_{1}, t\right) d s_{1}$. 
We integrate in $s$ to find $\dot{M}_{1}=M_{1}^{2}$, therefore the normalized density $f / M_{1}$ satisfies the equation

$$
\frac{1}{M_{1}} \partial_{t}\left(\frac{f}{M_{1}}\right)=Q\left(\frac{f}{M_{1}}, \frac{f}{M_{1}}\right) .
$$

Up to a change of time scale, this is a fundamental mean-field model of coalescence: Smoluchowski's coagulation equation with additive kernel. We treat this equation in greater depth below.

More precisely, it turns out that the random solution $u(x, t)$ has the structure described in (15) when the initial data $u_{0}$ consists of a compound Poisson process with only downward jumps. The mean drift rate at time $t$ is then $-M_{1}(t)$, and this example shows that the solution blows up at the time $M_{1}(0)^{-1}$ in this case. We show below (see (23)) that one may remove the mean drift by a change of scale and slope, yielding 'sawtooth' data with a deterministic upward drift that compensates the random downward jumps. For such data we obtain a global solution. Thus, there is no essential distinction between sawtooth data and the decreasing initial data considered above.

\subsection{The Cole-Hopf formula}

The modern notion of an entropy solution stems from the penetrating analysis by Hopf of the vanishing viscosity limit to (1). His work was based on a change of variables (re)discovered independently by Cole and Hopf [15, 27]. This solution is obtained via minimization of the Cole-Hopf function [15, 27]

$$
H(y, t ; x)=\frac{(x-y)^{2}}{2 t}+\int_{-\infty}^{y} u_{0}\left(y^{\prime}\right) d y^{\prime} .
$$

The minimum in $y$ is well-defined for all $t>0$ provided $U(y)=\int_{0}^{y} u_{0}\left(y^{\prime}\right) d y^{\prime}$ is lower semicontinuous and $\lim _{x \rightarrow \pm \infty} y^{-2} U(y)=0$. This is a mild assumption and holds for the random data we consider provided that the mean drift is zero. We denote the extreme points where $H$ is minimized by

$a_{-}(x, t)=\inf \left\{z \mid H(z, t ; x)=\min _{y} H\right\}, \quad a_{+}(x, t)=\sup \left\{z \mid H(z, t ; x)=\min _{y} H\right\}$.

Notice that any $z \in \mathbb{R}$ such that $x=t u_{0}(z)+z$ is a critical point of $H$, and represents a Lagrangian point that arrives at $x$ at time $t$. Of these $z$, the 'correct' Lagrangian points are the minimizers of $H$. If $a_{-}(x, t)=a_{+}(x, t)$, this point is unique, and we have

$$
u(x, t)=\frac{x-a_{ \pm}(x, t)}{t}, \quad x \in \mathbb{R}, t>0 .
$$


There is a shock at $(x, t)$ when $a_{-}(x, t) \neq a_{+}(x, t)$. In this case, the Lagrangian interval $\left[a_{-}(x, t), a_{+}(x, t)\right]$ is absorbed into the shock and the velocity of the shock is given by the Rankine-Hugoniot condition (conservation of momentum)

$$
u(x, t)=\frac{u\left(x_{+}, t\right)+u\left(x_{-}, t\right)}{2}=\frac{1}{a_{+}(x, t)-a_{-}(x, t)} \int_{a_{-}(x, t)}^{a_{+}(x, t)} u_{0}(y) d y .
$$

It will be convenient for us to assume that $u$ is right-continuous and we call $a(x, t)=a_{+}(x, t)$ the inverse Lagrangian function. Of course, the speed of shocks are still determined by the right-hand side of (21).

In order to deal with non-zero mean drift in initial data, we will use the following interesting invariance of Burgers equation. Assume that $u_{0}(x)=$ $o(|x|)$ as $|x| \rightarrow \infty$, and let $u(x, t)$ be the Cole-Hopf solution with $u(x, 0)=$ $u_{0}(x)$, defined for all $t \geq 0$. Let $c \in \mathbb{R}$ and define

$$
u_{0}^{(c)}(x)=u_{0}(x)+c x, \quad T_{c}=\left\{\begin{aligned}
-c^{-1}, & c<0 \\
+\infty, & c \geq 0
\end{aligned}\right.
$$

Then the Cole-Hopf solution with initial data $u_{0}^{(c)}$ is given by

$$
u^{(c)}(x, t)=\frac{1}{1+c t} u\left(\frac{x}{1+c t}, \frac{t}{1+c t}\right)+\frac{c x}{1+c t}, \quad t \in\left[0, T_{c}\right) .
$$

This is seen as follows. An elementary calculation shows that the Cole-Hopf functionals for the different data are related by

$$
H^{(c)}(y, t ; x)=H\left(y, \frac{t}{1+c t} ; \frac{x}{1+c t}\right)+\frac{c x^{2}}{2(1+c t)},
$$

which implies the inverse Lagrangian functions are related by

$$
a^{(c)}(x, t)=a\left(\frac{x}{1+c t}, \frac{t}{1+c t}\right) .
$$

We now substitute in (20) to obtain (23).

\subsection{Solutions with Lévy process initial data}

Here we describe how the solution of (1), with initial data of the form (3), is determined in terms of Laplace exponents, essentially following Bertoin's treatment in $[5]$. 
Suppose $x \mapsto u_{0}(x)$ is an arbitrary spectrally negative Lévy process for $x \geq 0$, with Laplace exponent $\psi_{0}$ having downward jump measure $\Lambda_{0}$. We first show that we may assume without loss of generality that $\int_{0}^{\infty}(s \wedge$ $\left.s^{2}\right) \Lambda_{0}(d s)<\infty$. Indeed, if $\int_{1}^{\infty} s \Lambda_{0}(d s)=\infty$, then $u_{0}(x) / x \rightarrow-\infty$ almost surely as $x \rightarrow \infty$. (This follows from the fact that for the compound Poisson process $X_{x}$ with jump measure $\Lambda_{0}(d s) \mathbf{1}_{|s| \geq 1}$, one has $X_{x} / x \rightarrow \infty$ as $x \rightarrow \infty$ by the law of large numbers.) In this case the Cole-Hopf function $H(y, t ; x)$ has no minimum for any $t>0$, and equation (1) has no finite entropy solution for any positive time. Hence, we may suppose that $\int_{1}^{\infty} s \Lambda_{0}(d s)<$ $\infty$.

Next, we show that one may assume the mean drift $b_{0}=\mathbb{E}\left(u_{0}(1)\right)$ is zero. If $b_{0}$ is nonzero, we have $\lim _{x \rightarrow \infty} u_{0}(x) / x=b_{0}$ a.s. by the strong law of large numbers. If $b_{0}<0$, then by comparison to compression-wave solutions with initial data $A+b \max (x, 0)$, we find using the maximum principle that a.s. the solution blows up exactly at time $-b_{0}^{-1}$. If $b_{0}>0$ there is a global solution. In either case, we may use the transformation (23) with $c=b_{0}$ to reduce to the case $b_{0}=0$, replacing $u_{0}(x)$ by $u_{0}(x)-b_{0} x \mathbf{1}_{x>0}$. More precisely, we apply (23) for $x \geq 0$ noting that $a(0, t) \geq 0$, thus $a(x, t) \geq a(0, t) \geq 0$ for $x \geq 0$, so that (24) holds for $x \geq 0$. We have:

Lemma 1. If $u_{0}^{(c)}$ is a spectrally negative Lévy process with Lévy triplet $\left(c, \sigma_{0}^{2}, \Lambda_{0}\right)$, the Cole-Hopf solution $u^{(c)}(x, t)$ is determined via (23) for $x \geq 0$ and $t \in\left[0, T_{c}\right)$, in terms of a solution $u(x, t)$ having zero mean drift and defined for all $t \geq 0$.

With these reductions, we may restrict ourselves to Laplace exponents $\psi_{0}$ of the form

$$
\psi_{0}(q)=\frac{\sigma_{0}^{2} q^{2}}{2}+\int_{0}^{\infty}\left(e^{-q s}-1+q s\right) \Lambda_{0}(d s), \quad q \geq 0 .
$$

We will always assume that $a$ and $u$ are right continuous (i.e., $a(x, t)=$ $a\left(x_{+}, t\right)$, compare with (21)). This ensures $a$ is an element of the Skorokhod space $\mathbb{D}$, so that we may use the standard Skorokhod topology to study limiting behavior. For brevity, we write

$$
v(x, t)=u(x, t)-u(0, t), \quad l(x, t)=a(x, t)-a(0, t),
$$

and rewrite (20) as

$$
v(x, t)=\frac{x-l(x, t)}{t}, \quad x \geq 0, t>0
$$


Bertoin has shown that for all $t>0, x \mapsto l(x, t)$ is an increasing Lévy process (a subordinator) with the same law as the first passage process for $t u_{0}(x)+x$. We denote the Laplace exponents of $l$ and $v$ by $\Phi$ and $\psi$ respectively:

$$
\mathbb{E}\left(e^{-q l(x, t)}\right)=e^{-x \Phi(q, t)}, \quad \mathbb{E}\left(e^{q v(x, t)}\right)=e^{x \psi(q, t)}, \quad x, q, t \geq 0 .
$$

We combine (26) and (27) to obtain

$$
\psi(q, t)=\frac{q}{t}-\Phi\left(\frac{q}{t}, t\right) .
$$

Since $l$ is a subordinator, it has the simpler Lévy-Khintchine representation

$$
\Phi(q, t)=d_{t} q+\int_{0}^{\infty}\left(1-e^{-q s}\right) \mu_{t}(d s), \quad q>0,
$$

where $d_{t} \geq 0$ supplies the deterministic part of the drift, and $\mu_{t}$ is the Lévy measure of $l(\cdot, t)$, which now must satisfy $\int_{0}^{\infty}(1 \wedge s) \mu_{t}(d s)<\infty[4]$. We see from $(26)$ that $v(\cdot, t)$ is a Lévy process with no Gaussian component, and thus has a Lévy-Khintchine representation

$$
\psi(q, t)=b_{t} q+\int_{0}^{\infty}\left(e^{-q s}-1+q s\right) \Lambda_{t}(d s), \quad q \geq 0, t>0,
$$

related to (29) by

$$
\Lambda_{t}(d s)=\mu_{t}(t d s), \quad b_{t}+\int_{0}^{\infty} s \Lambda_{t}(d s)=\frac{1-d_{t}}{t} .
$$

Due to the result that $l$ and the first passage process of $t u_{0}(x)+x$ have the same law, a simple functional relation holds between $\psi_{0}$ and $\Phi(q, t)[5$, Thm. 2]:

$$
\psi_{0}(t \Phi(q, t))+\Phi(q, t)=q, \quad q \geq 0, t>0 .
$$

The evolution takes a remarkably simple form when we combine equations (28) and (32) to obtain

$$
\psi(q, t)=\psi_{0}(q-t \psi(q, t)), \quad q \geq 0, t>0 .
$$

But then $\psi(q, t)$ solves the inviscid Burgers equation (in $q$ and $t$ !)

$$
\partial_{t} \psi+\psi \partial_{q} \psi=0, \quad \psi(0, q)=\psi_{0}(q) .
$$

The solution to (34) may be constructed by the method of characteristics and takes the form (33). The remarkable fact that the Laplace exponent 
is also a solution to Burgers equation was first observed by Carraro and Duchon [12, Thm. 2].

Since $\psi_{0}$ is analytic and strictly convex, the solution (33) is analytic for all time and unique, and the condition $\partial_{q} \psi(0, t)=0$ is preserved for all $t>0$. By (28)-(31), we have

$$
b_{t}=0, \quad d_{t}=1-t \int_{0}^{\infty} s \Lambda_{t}(d s), \quad t>0 .
$$

Let

$$
M_{0}=\lim _{q \rightarrow \infty} \psi_{0}^{\prime}(q),
$$

We find $\Phi(q, t) \rightarrow \infty$ as $q \rightarrow \infty$ from (32), and differentiate to obtain

$$
d_{t}=\lim _{q \rightarrow \infty} \partial_{q} \Phi(q, t)=\lim _{q \rightarrow \infty} \frac{1}{1+t \psi_{0}^{\prime}(t \Phi(q, t))}=\frac{1}{1+t M_{0}}, \quad t>0,
$$

with the understanding that $d_{t}=0$ if $M_{0}=+\infty$. Then

$$
M(t):=\lim _{q \rightarrow \infty} \partial_{q} \psi(q, t)=\int_{0}^{\infty} s \Lambda_{t}(d s)=\frac{M_{0}}{1+t M_{0}}, \quad t>0,
$$

with the understanding that $M(t)=t^{-1}$ when $M_{0}=\infty$. Note $M^{\prime}=-M^{2}$. Below we will characterize the evolution of $\Lambda_{t}$ differently.

\subsection{BV regularity}

It is clear from the Cole-Hopf formula that $u$ is locally of bounded variation for every $t>0$. We derive a decay estimate that quantifies this. The sample paths of $u_{0}$ have unbounded variation if and only if $[4, \mathrm{p} .15]$

$$
\sigma_{0}^{2}>0 \quad \text { or } \quad \int_{0}^{\infty} s \Lambda_{0}(d s)=\infty
$$

Heuristically, this corresponds to the presence of many small jumps ('dust'). This is reflected in the Laplace exponent as $M_{0}=\lim _{q \rightarrow \infty} \psi_{0}^{\prime}(q)=+\infty$ in this case. On the other hand, $M_{0}$ is finite if and only if $u_{0}$ is BV, in which case $\sigma_{0}=0$ and $M_{0}=\int_{0}^{\infty} s \Lambda_{0}(d s)<\infty$.

The analytic formula (29) has the following probabilistic meaning. If we take a Poisson point process $x \mapsto m_{x}^{t}$ (masses of clusters) with jump measure $\mu_{t}$ we have the representation $[4, \mathrm{p} .16]$

$$
l(x, t)=d_{t} x+\sum_{0 \leq y \leq x} m_{y}^{t} .
$$


The velocity field, and a point process of shock strengths $s_{y}^{t}=t^{-1} m_{y}^{t}$ are determined from (20), (37) and (40) by

$$
v(x, t)=M(t) x-\frac{1}{t} \sum_{0 \leq y \leq x} m_{y}^{t}=M(t) x-\sum_{0 \leq y} s_{y}^{t},
$$

For every $t>0, v(x, t)$ is the difference of two increasing functions: a linear drift and a pure jump process. Thus, it is of bounded variation, and by (35) and (40) we have

$$
\mathbb{E}\left(\int_{0}^{x}\left|\partial_{y} v(y, t)\right| d y\right)=2 M(t) x, \quad x, t>0,
$$

because $\mathbb{E}\left(\sum_{0 \leq y \leq x} m_{y}^{t}\right)=x M(t)=x\left(1-d_{t}\right)$.

\subsection{Relation to Smoluchowski's coagulation equation}

We consider a positive measure $\nu_{\tau}(d s)$ interpreted as the number of clusters of mass or size $s$ per unit volume at time $\tau$. Clusters of mass $r$ and $s$ coalesce by binary collisions at a rate governed by a symmetric kernel $K(r, s)$. A weak formulation of Smoluchowski's coagulation equation can be based on a general moment identity for suitable test functions $\zeta$ (see [32]):

$$
\begin{aligned}
\partial_{\tau} \int_{0}^{\infty} \zeta(s) \nu_{\tau}(d s)= \\
\quad \frac{1}{2} \int_{0}^{\infty} \int_{0}^{\infty}(\zeta(r+s)-\zeta(r)-\zeta(s)) K(r, s) \nu_{\tau}(d r) \nu_{\tau}(d s) .
\end{aligned}
$$

We consider only the additive kernel $K(r, s)=r+s$. It is classical that (43) can then be solved by the Laplace transform [16]. We denote the initial time by $\tau_{0}$ (to be chosen below). The minimal (and natural) hypothesis on initial data $\nu_{\tau_{0}}$ is that the mass $\int_{0}^{\infty} s \nu_{\tau_{0}}(d s)$ is finite. We scale the initial data such that $\int_{0}^{\infty} s \nu_{\tau_{0}}=1$. The Laplace exponent

$$
\varphi(q, \tau)=\int_{0}^{\infty}\left(1-e^{-q s}\right) \nu_{\tau}(d s)
$$

then satisfies

$$
\partial_{\tau} \varphi-\varphi \partial_{q} \varphi=-\varphi, \quad \tau>\tau_{0} .
$$

We showed in [32] that (45) may be used to define unique, global, masspreserving solutions to (43). In particular, a map $\tau \mapsto \nu_{\tau}$ from $\left[\tau_{0}, \infty\right)$ to 
the space of positive Radon measures on $(0, \infty)$, such that $\int_{0}^{\infty} s \nu_{\tau}(d s)=1$ for all $\tau \geq \tau_{0}$, is a solution of Smoluchowski's equation with $K(r, s)=r+s$ (in an appropriate weak sense detailed in [32]) if and only if $\varphi$ satisfies (45).

We now connect solutions of the inviscid Burgers equation (1) with Lévy process initial data to solutions of Smoluchowski's equation through a change of scale. Let $u_{0}$ satisfy (3) and assume as in subsection 2.3 that the corresponding downward jump measure $\Lambda_{0}$ satisfies $\int_{0}^{\infty}\left(s \wedge s^{2}\right) \Lambda_{0}(d s)<\infty$ and the mean drift is zero. Let $\Lambda_{t}$ be the jump measure of the Cole-Hopf solution. With $M_{0}$ and $M(t)$ as in (36) and (38), let $\tau_{0}=-\log M_{0}$ if $u_{0}$ is of bounded variation, and $\tau_{0}=-\infty$ otherwise, and set

$$
\tau=-\log M(t), \quad \nu_{\tau}(d s)=\Lambda_{t}(M(t) d s) .
$$

From (38) it follows $\int_{0}^{\infty} s \nu_{\tau}(d s)=1$, and by (44) and (30) we find

$$
\varphi(q, \tau)=q-\psi\left(q e^{\tau}, t\right)
$$

We see that $\psi$ solves (34) if and only if $\varphi$ solves (45). Therefore, the rescaled Lévy measure of $v(\cdot, t)$ evolves according to Smoluchowski's equation. Conversely, given any solution of Smoluchowski's equation with initial data $\nu_{0}$ at a finite $\tau_{0}$, we can construct a corresponding solution of (1) by choosing $u_{0}$ to be a spectrally negative Lévy process with jump measure $\Lambda_{t_{0}}$ via (46)

Initial data $u_{0}$ with unbounded variation are of particular interest. Here we have eternal solutions $\nu_{\tau}$ to (43) defined for all $\tau \in \mathbb{R}$. We see that eternal solutions are in one-to-one correspondence with initial data $u_{0}$ of unbounded variation via (47). A finer correspondence mapping the clustering of shocks to the additive coalescent is found in [6].

To summarize, we have the following correspondence.

Theorem 2. Assume $u_{0}$ is a spectrally negative Lévy process with Lévy triplet $\left(0, \sigma_{0}^{2}, \Lambda_{0}\right)$, with the same assumptions as in Theorem 1. Then for all $t>0, v(\cdot, t)$ and $l(\cdot, t)$ are Lévy processes with triplet $\left(0,0, \Lambda_{t}\right)$, whose jump measures $\Lambda_{t}$ determine a solution $\nu_{\tau}(d s)$ to Smoluchowski's coagulation equation with rate kernel $K(r, s)=r+s$ as described in (46).

\subsection{Self-similar solutions}

Bertoin's characterization of eternal solutions is the analogue of the LévyKhintchine characterization of infinitely divisible distributions [4, 18]. Among the latter, the stable distributions are of particular interest, and their analogues for Smoluchowski's equations are obtained by choosing the Laplace 
exponent $\psi_{0}(q)=q^{\alpha}, \alpha \in(1,2]$. For $\alpha \in(1,2)$ the corresponding Lévy measures are

$$
\Lambda(d s)=\frac{s^{-(1+\alpha)}}{\Gamma(-\alpha)} d s .
$$

The Laplace exponent $q^{2}$ corresponds to an atom at the origin. We thereby obtain for $\alpha \in(1,2]$ a family of self-similar solutions to Smoluchowski's equation with Laplace exponent of the form $\varphi(\tau, q)=e^{-\beta \tau} \varphi_{\alpha}\left(q e^{\beta \tau}\right)$ where $\varphi_{\alpha}$ solves

$$
\varphi_{\alpha}(q)^{\alpha}+\varphi_{\alpha}(q)=q, \quad q>0 .
$$

The self-similar solutions to Smoluchowski's coagulation equation are

$$
\nu_{\tau}(d s)=e^{-2 \tau / \beta} f_{\alpha}\left(e^{-\tau / \beta} s\right) d s, \quad \beta=\frac{\alpha-1}{\alpha}, \quad \alpha \in(1,2],
$$

where $f_{\alpha}$ has been defined in (7). An analytic proof that these are the only self-similar solutions to Smoluchowski's equation may be found in [32]. Each of these solutions corresponds to a self-similar process. Precisely, let $X^{\alpha}$ denote the stable process with Laplace exponent $q^{\alpha}$, and $T^{\alpha}$ and $V^{\alpha}$ denote the processes

$$
T_{x}^{\alpha}=\inf \left\{y \geq 0: X_{y}^{\alpha}+y>x\right\}, \quad V_{x}^{\alpha}=x-T_{x}^{\alpha} .
$$

We have $M_{0}=+\infty$ and $M(t)=t^{-1}=e^{-\tau}$ in this case, and the Laplace exponent of the process $l(\cdot, t)$ is of the self-similar form

$$
\Phi(q, t)=\varphi(q, \tau)=t^{-1 / \beta} \varphi_{\alpha}\left(q t^{1 / \beta}\right), t>0 .
$$

The solution processes have the scaling property

$$
l(x, t) \stackrel{\mathcal{L}}{=} t^{1 / \beta} T_{x t^{-1 / \beta}}^{\alpha}, \quad v(x, t) \stackrel{\mathcal{L}}{=} t^{1 / \beta-1} V_{x t^{-1 / \beta}}^{\alpha} .
$$

The corresponding Lévy measures are obtained from (31), (46) and (49):

$$
\mu_{t}^{\alpha}(d s)=t^{-2 / \beta} f_{\alpha}\left(t^{-1 / \beta} s\right) d s, \quad \Lambda_{t}^{\alpha}(d s)=t^{1-2 / \beta} f_{\alpha}\left(t^{1-1 / \beta} s\right) d s .
$$

In the important case $\alpha=2$, we have $1 / \beta=2$ and $\varphi_{2}(q)=-\frac{1}{2}+\sqrt{\frac{1}{4}+q}$, and by Laplace inversion [1, Ch.29] we obtain the explicit expression in (11) for the distribution of $T_{x}^{2}$. 


\section{The convergence theorem}

In [32] we proved the following theorem characterizing solutions that approach self-similar form in Smoluchowski's coagulation equation with additive kernel. To every solution $\nu_{\tau}$ of (43) with $\int_{0}^{\infty} s \nu_{\tau}(d s)=1$ we associate the probability distribution function

$$
F(s, \tau)=\int_{(0, s]} r \nu_{\tau}(d r)
$$

To a self-similar solution $f_{\alpha}, \alpha \in(1,2]$ with $\beta=(\alpha-1) / \alpha$ we associate

$$
F_{\alpha}(s)=\int_{0}^{s} r f_{\alpha}(r) d r=\sum_{k=1}^{\infty} \frac{(-1)^{k-1} s^{k \beta}}{k !} \Gamma(1+k-k \beta) \frac{\sin \pi k \beta}{\pi k \beta} .
$$

A probability distribution function $F^{*}$ is called nontrivial if $F^{*}(s)<1$ for some $s>0$; this means the distribution is proper $\left(\lim _{s \rightarrow \infty} F^{*}(s)=1\right)$ and not concentrated at 0 .

Theorem 3. Suppose $\tau_{1} \in \mathbb{R}$ and $\nu_{\tau}, \tau \in\left[\tau_{1}, \infty\right)$, is a solution to Smoluchowski's coagulation equation with additive kernel such that $\int_{0}^{\infty} s \nu_{\tau_{1}}(d s)=$ 1.

1. Suppose there is a rescaling function $\tilde{\lambda}(\tau) \rightarrow \infty$ as $\tau \rightarrow \infty$ and a nontrivial probability distribution function $F^{*}$ such that

$$
\lim _{\tau \rightarrow \infty} F(\tilde{\lambda}(\tau) s, \tau)=F^{*}(s)
$$

at all points of continuity of $F^{*}$. Then there exists $\alpha \in(1,2]$ and a function $L$ slowly varying at infinity such that

$$
\int_{0}^{s} r^{2} \nu_{\tau_{1}}(d r) \sim s^{2-\alpha} L(s) \quad \text { as } s \rightarrow \infty
$$

2. Conversely, assume that there exists $\alpha \in(1,2]$ and a function $L$ slowly varying at infinity such that (57) holds. Then there is a strictly increasing rescaling $\tilde{\lambda}(\tau) \rightarrow \infty$ such that

$$
\lim _{\tau \rightarrow \infty} F(\tilde{\lambda}(\tau) s, \tau)=F_{\alpha}(s), \quad 0 \leq s<\infty,
$$

where $F_{\alpha}$ is a distribution function for a self similar solution as in (55). 
We now prove Theorem 1 . Let $u_{0}$ be a spectrally negative Lévy process with zero mean drift and $\int_{0}^{\infty}\left(s \wedge s^{2}\right) \Lambda_{0}(d s)<\infty$. To the solution increment $v(x, t)=u(x, t)-u(0, t)$ with downward jump measure $\Lambda_{t}$, associate a solution $\nu_{\tau}$ of Smoluchowski's coagulation equation (43) as in Theorem 2 with Laplace exponent $\varphi(q, \tau)$ given by (47). Let $\tau_{1}=\tau_{0}=-\log M_{0}$ if $M_{0}<\infty$, and let $\tau_{1}=0$ if $M_{0}=+\infty$ and $\tau_{0}=-\infty$.

We deduce Theorem 1 from Theorem 3 by establishing two equivalences:

(a) There is a rescaling $\lambda(t) \rightarrow \infty$ as $t \rightarrow \infty$ and a Lévy process $V^{*}$ with zero mean drift $\mathbb{E}\left(V^{*}\right)$ such that $V^{(t)} \stackrel{\mathcal{L}}{\rightarrow} V^{*}$ if and only if there is a rescaling $\tilde{\lambda}(\tau) \rightarrow \infty$ as $\tau \rightarrow \infty$ and a nontrivial probability distribution function $F^{*}$ such that (56) holds.

(b) $\int_{0}^{\infty} s^{2} \nu_{\tau_{1}}(d s)<\infty$ if and only if $\int_{0}^{\infty} s^{2} \Lambda_{0}(d s)<\infty$. Moreover, $\int_{0}^{s} r^{2} \nu_{\tau_{1}}(d r) \sim$ $s^{2-\alpha} L(s)$ as $s \rightarrow \infty$ if and only if $\int_{0}^{s} r^{2} \Lambda_{0}(d r) \sim s^{2-\alpha} L(s)$ as $s \rightarrow \infty$.

Proof of (a). We prove claim (a) by showing each part equivalent to a corresponding convergence statement for rescaled Laplace exponents. First, convergence in law in $\mathbb{D}$ for processes with independent increments can be reduced to the convergence of characteristic exponents [28, Cor. VII.4.43, p.440]. In particular, suppose $\lambda(t) \rightarrow \infty$ as $t \rightarrow \infty$. Then we have

$$
V^{(t)} \stackrel{\mathcal{L}}{\rightarrow} V^{*}, \quad \text { with } \mathbb{E}\left(V_{1}^{*}\right)=0
$$

if and only if $\mathbb{E}\left(e^{i k V_{x}^{(t)}}\right) \rightarrow \mathbb{E}\left(e^{i k V_{x}^{*}}\right)$ for all $k \in \mathbb{R}$, uniformly for $x$ in compact sets, and $\mathbb{E}\left(V_{1}^{*}\right)=0$. But since we are working with Lévy processes, the Lévy-Khintchine formula shows the dependence on $x$ is trivial, and thus (58) is equivalent to

$$
\mathbb{E}\left(e^{i k V_{1}^{(t)}}\right) \rightarrow \mathbb{E}\left(e^{i k V_{1}^{*}}\right) \quad \text { for all } k \in \mathbb{R}, \quad \text { and } \mathbb{E}\left(V_{1}^{*}\right)=0 .
$$

But pointwise convergence of characteristic functions is equivalent to convergence in distribution of the random variables $V_{1}^{(t)}[18, \mathrm{XV} .3 .2]$, and since $V_{1}^{(t)}=1-T_{1}^{(t)} \leq 1,(59)$ is equivalent to convergence of the Laplace transforms [18, XIII.1.2]:

$$
\mathbb{E}\left(e^{q V_{1}^{(t)}}\right) \rightarrow \mathbb{E}\left(e^{q V_{1}^{*}}\right) \text { for all } q>0, \text { and } \mathbb{E}\left(V_{1}^{*}\right)=0 .
$$

Taking logarithms and using (12) and (27), (60) is equivalent to

$$
\lambda \psi(q t / \lambda, t) \rightarrow \psi^{*}(q) \text { for all } q>0, \quad \text { and } \partial_{q} \psi^{*}(0)=0,
$$


where $\mathbb{E}\left(e^{q V_{x}^{*}}\right)=e^{x \psi^{*}(q)}$. This expresses the convergence of $V^{(t)}$ in terms of convergence of rescaled Laplace exponents.

Now suppose $\tilde{\lambda}(\tau) \rightarrow \infty$ as $\tau \rightarrow \infty$. Using [18, XIII.1.2] again, the (proper) convergence in (56) is equivalent to pointwise convergence of Laplace transforms:

$$
\eta(q, \tau) \rightarrow \eta^{*}(q) \text { for all } q>0, \text { with } \eta^{*}(0)=1 .
$$

where $\eta(q, \tau):=\int_{0}^{\infty} e^{-q s} F(\tilde{\lambda}(\tau) d s, \tau), \eta^{*}(q):=\int_{0}^{\infty} e^{-q s} F^{*}(d s)$. By (54) and (44), we have

$$
\eta(q, \tau)=\left(\partial_{q} \varphi\right)(q / \tilde{\lambda}, \tau), \quad \int_{0}^{q} \eta(r, \tau) d r=\tilde{\lambda} \varphi(q / \tilde{\lambda}, \tau) .
$$

We claim that $(62)$ is equivalent to the statement that (with $\varphi^{*}(q)=$ $\left.\int_{0}^{q} \eta^{*}(r) d r\right)$

$$
\tilde{\lambda} \varphi(q / \tilde{\lambda}, \tau) \rightarrow \varphi^{*}(q) \text { for all } q>0, \quad \text { and } \partial_{q} \varphi^{*}(0)=1 .
$$

Clearly, since $\eta(\cdot, \tau)$ is completely monotone and bounded, (62) implies (64). In the other direction, assume (64). For any sequence $\tau_{j} \rightarrow \infty$ there is a subsequence along which $\eta\left(q, \tau_{j}\right)$ converges for all (rational, hence real) $q>0$, to some limit whose integral must be $\varphi^{*}$. Thus (62) follows.

We now finish the proof of claim (a) by observing that due to (47), we have

$$
\tilde{\lambda} \varphi(q / \tilde{\lambda}, \tau)=q-\tilde{\lambda} \psi\left(q e^{\tau} / \tilde{\lambda}, t\right) .
$$

Hence the convergence in (61) is equivalent to that in (64) provided we have

$$
\lambda(t) / t=\tilde{\lambda}(\tau) / e^{\tau}
$$

or $\lambda(t)=t M(t) \tilde{\lambda}(\tau)$, since $t M(t) \rightarrow 1$ as $t \rightarrow \infty$. (Note $t M(t)=1$ if $M_{0}=\infty$.)

Proof of (b). It is only the case $M_{0}=\infty$ that requires some work. Indeed, if $M_{0}<\infty$ we see from (46) that $\nu_{\tau_{1}}(d s)=\Lambda_{0}\left(M_{0} d s\right)$. In what follows, we suppose that $M_{0}=\infty$. We then have an eternal solution to Smoluchowski's equation, and $t=e^{\tau}$. We shall compare the tails of $\nu_{0}(\tau=0)$ with that of $\Lambda_{0}(t=0)$.

Claim (b) is a purely analytic fact that follows from Karamata's Tauberian theorem [18]. We first reformulate it as a statement about Laplace transforms. Let $\varphi_{0}(q)=\varphi(q, 0), \psi_{0}(q)=\psi(q, 0)$. For every $\alpha \in(1,2]$ we have

$$
\int_{0}^{s} r^{2} \nu_{0}(d r) \sim s^{2-\alpha} L(s) \Longleftrightarrow 1-\varphi_{0}^{\prime}(q) \sim q^{\alpha-1} L\left(\frac{1}{q}\right) \frac{\Gamma(3-\alpha)}{\alpha-1}
$$


as $s \rightarrow \infty$ and $q \rightarrow 0$ respectively (see [32, eq. 7.4]). By the same argument,

$$
\int_{0}^{s} r^{2} \Lambda_{0}(d r) \sim s^{2-\alpha} L(s) \Longleftrightarrow \psi_{0}^{\prime}(q) \sim q^{\alpha-1} L\left(\frac{1}{q}\right) \frac{\Gamma(3-\alpha)}{\alpha-1},
$$

with the following caveat when $\alpha=2$. If $\int_{0}^{\infty} r^{2} \Lambda(d r)=\infty$ then (68) holds. On the other hand, if $\int_{0}^{\infty} r^{2} \Lambda_{0}(d r)<\infty$ then we must modify the second condition in (68) to

$$
\psi_{0}^{\prime}(q) \sim\left(\sigma^{2}+\int_{0}^{\infty} r^{2} \Lambda(d r)\right) q, \quad q \rightarrow 0 .
$$

We set $t=1$ in (47) and differentiate (33) with respect to $q$ to obtain

$$
\psi_{0}^{\prime}\left(\varphi_{0}(q)\right)=\frac{1-\varphi_{0}^{\prime}(q)}{\varphi_{0}^{\prime}(q)}=\frac{1}{\varphi_{0}^{\prime}(q)}-1 .
$$

The functions $\psi_{0}^{\prime}, \varphi_{0}$, and $1 / \varphi^{\prime}$ are strictly increasing. Since $\varphi_{0}^{\prime}(0)=1$ we also have $\varphi_{0}(q)=q(1+o(1))$ as $q \rightarrow 0$. A sandwich argument as in [18] may now be used to deduce claim (b). First suppose that (67) holds. Fix $b, \varepsilon>0$. Then for $q$ sufficiently small we use monotonicity and (69) to obtain

$$
\frac{1-\varphi_{0}^{\prime}(b q(1-\varepsilon))}{1-\varphi_{0}^{\prime}(q(1+\varepsilon))} \frac{\varphi_{0}^{\prime}(q(1+\varepsilon))}{\varphi_{0}^{\prime}(b q(1-\varepsilon))}<\frac{\psi_{0}^{\prime}(b q)}{\psi_{0}^{\prime}(q)}<\frac{1-\varphi_{0}^{\prime}(b q(1+\varepsilon))}{1-\varphi_{0}^{\prime}(q(1-\varepsilon))} \frac{\varphi_{0}^{\prime}(q(1-\varepsilon))}{\varphi_{0}^{\prime}(b q(1+\varepsilon))} .
$$

Letting first $q$ and then $\varepsilon \rightarrow 0$, we obtain

$$
\lim _{q \rightarrow 0} \frac{\psi_{0}^{\prime}(b q)}{\psi_{0}^{\prime}(q)}=b^{\alpha-1}
$$

Thus, $\psi_{0}^{\prime}$ is regularly varying with exponent $\alpha-1$. Similarly, if we assume that (68) holds, we sandwich

$$
\frac{\psi_{0}^{\prime}(b(1-\varepsilon) q)}{\psi_{0}^{\prime}((1+\varepsilon) q)}<\frac{1-\varphi_{0}^{\prime}(b q)}{1-\varphi_{0}^{\prime}(q)} \frac{\varphi_{0}^{\prime}(q)}{\varphi_{0}^{\prime}(b q)} \cdot<\frac{\psi_{0}^{\prime}(b(1+\varepsilon) q)}{\psi_{0}^{\prime}((1-\varepsilon) q)}
$$

to deduce that $1-\varphi_{0}^{\prime}$ is regularly varying with exponent $\alpha-1$. Finally, since $\varphi_{0}^{\prime}(0)=1$ it follows from $(69)$ that $\lim _{q \rightarrow 0} \psi_{0}^{\prime}(q) /\left(1-\varphi_{0}^{\prime}(q)\right)=1$. This finishes the proof of Theorem 1.

\section{Energy, dissipation and spectra}

In this section, we compute several statistics of physical interest for the solution increments: mean energy and dissipation, the law of the FourierLaplace transform, and the multifractal spectrum. While the computations 
are routine, some interesting features emerge, namely (i) conservation of energy despite dissipation at shocks, (ii) a simple evolution rule for the Fourier-Laplace spectrum, and (iii) a multifractal spectrum in sharp variance with that of fully developed turbulence. Simple proofs of fine regularity properties (e.g., Hausdorff dimension of the set of Lagrangian regular points) may be found in [5].

\subsection{Energy and dissipation}

The energy in any finite interval $I \subset \mathbb{R}_{+}$is computed using the LévyKhintchine formula (30) and Fubini's theorem as follows.

$$
\begin{aligned}
& \mathbb{E}\left(\int_{I} v(x, t)^{2} d x\right)=\int_{I} \mathbb{E}\left(v(x, t)^{2}\right) d x=\int_{I}\left(\left.\partial_{q}^{2} \mathbb{E}\left(e^{q v(x, t)}\right)\right|_{q=0}\right) d x \\
& =\left.\int_{I} \partial_{q}^{2} e^{x \psi(q, t)}\right|_{q=0} d x=\int_{I}\left(x^{2} \partial_{q} \psi(0, t)^{2}+x \partial_{q}^{2} \psi(0, t)\right) d x \\
& =b_{t}^{2} \int_{I} x^{2} d x+\left(\int_{0}^{\infty} y^{2} \Lambda_{t}(d y)\right) \int_{I} x d x
\end{aligned}
$$

Let us restrict attention to solutions of mean zero, that is $b_{t}=0$. Then we have conservation of energy in the sense that

$$
\mathbb{E}\left(\int_{I} v(x, t)^{2} d x\right)=\mathbb{E}\left(\int_{I} v(x, 0)^{2} d x\right), \quad t \geq 0 .
$$

Indeed, by (70), we see that (71) is equivalent to

$$
\partial_{q}^{2} \psi(0, t)=\partial_{q}^{2} \psi_{0}=\sigma_{0}^{2}+\int_{0}^{\infty} s^{2} \Lambda_{0}(d s)=: M_{2},
$$

with the understanding that $\partial_{q}^{2} \psi(0, t)=\infty$ if $\int_{0}^{\infty} s^{2} \Lambda_{0}(d s)$ is divergent. It is only necessary to differentiate (33) to obtain

$$
\partial_{q} \psi(q, t)=\frac{\psi_{0}^{\prime}(q-t \psi)}{1+t \psi_{0}^{\prime}(q-t \psi)}, \quad \partial_{q}^{2} \psi(q, t)=\frac{1}{\left(1+t \psi_{0}^{\prime}(q-t \psi)\right)^{3}} \psi_{0}^{\prime \prime}(q-t \psi),
$$

and then take the limit $q \rightarrow 0$ to obtain (71).

The dissipation at a shock with left and right limits $u_{ \pm}$is obtained as follows. The decay of the $L^{2}$ norm for solutions to Burgers equations with viscosity $\varepsilon, u_{t}+u u_{x}=\varepsilon u_{x x}$, is given by

$$
\frac{d}{d t} \int_{\mathbb{R}} u^{2} d x=2 \varepsilon \int_{\mathbb{R}} u_{x}^{2} d x .
$$


The right hand side may be evaluated exactly for traveling waves (viscous shocks) of the form $u(x, t)=u^{\varepsilon}(x-c t)$. It is easily seen that for any $\varepsilon>0$ a traveling wave profile connecting the states $u_{-}>u_{+}$at $\mp \infty$ is of the form $u^{\varepsilon}(x-c t)=w((x-c t) / \varepsilon)$ where $w$ satisfies the ordinary differential equation

$$
-c\left(w-u_{-}\right)+\frac{1}{2}\left(w^{2}-u_{-}^{2}\right)=\frac{d w}{d \xi}, \quad c=\frac{u_{-}+u_{+}}{2} .
$$

We therefore have

$$
\begin{aligned}
& 2 \varepsilon \int_{\mathbb{R}} u_{x}^{2} d x=2 \int_{\mathbb{R}}\left(w^{\prime}\right)^{2} d \xi=2 \int_{u_{-}}^{u_{+}}\left[-c\left(w-u_{-}\right)+\frac{1}{2}\left(w^{2}-u_{-}^{2}\right)\right] d w \\
& =2\left(u_{-}-u_{+}\right)^{3} \int_{0}^{1} w(1-w) d w=\frac{\left(u_{-}-u_{+}\right)^{3}}{3} .
\end{aligned}
$$

The right hand side is independent of $\varepsilon$ and captures the dissipation of the entropy solution in the limit $\varepsilon \rightarrow 0$. The dissipation at shocks in any finite interval $I \subset \mathbb{R}_{+}$may now be computed by summing over all shocks in $I$ using (40) and (41):

$$
\frac{1}{3} \mathbb{E}\left(\sum_{y \in I}\left(v\left(y_{-}, t\right)-v\left(y_{+}, t\right)\right)^{3}\right)=\frac{1}{3} \mathbb{E}\left(\sum_{y \in I}\left(s_{y}^{t}\right)^{3}\right)=\frac{|I|}{3} \int_{0}^{\infty} s^{3} \Lambda_{t}(d s),
$$

where $|I|$ is the length of $I$.

Conservation of energy in the sense described in (71) is rather surprising in view of the dissipation at shocks. In particular, there are solutions with finite energy $\left(\int_{0}^{\infty} s^{2} \Lambda_{t}(d s)<\infty\right)$, but infinite dissipation $\left(\int_{0}^{\infty} s^{3} \Lambda_{t}(d s)=\right.$ $\infty)$. However, there is no contradiction, since (71) refers to the expected value of the energy in any finite interval $I$, and the energy dissipated in shocks is compensated by energy input from the endpoints of $I$.

\subsection{The Fourier-Laplace spectrum}

We show that the law of the Fourier transform $\hat{v}(k, t)$ of paths $x \mapsto v(x, t)$, is determined by a Lévy process with jump measure $s^{-1} \bar{\Lambda}_{t}(s) d s$, where $\bar{\Lambda}_{t}(s)=\int_{s}^{\infty} \Lambda_{t}(d s)$. Here $\Lambda_{t}(d s)$ denotes the jump measure of $v(x, t)$ (see Theorem 4 below). The assertion $\hat{v} \sim k^{-1}$ as $k \rightarrow \infty$ for white noise initial data is common in the Burgers turbulence literature (e.g., see [20, 36]). For the present case of Lévy process initial data, we show that $\hat{v}(k, t) \sim$ $-i M(t) k^{-2}$ as $k \rightarrow \infty$. In addition, we find precise corrections under additional assumptions on $\Lambda_{t}$ (for example, for self-similar solutions). 
These computations with the laws of the Fourier-Laplace transform should be contrasted with the conventional notion of the power spectrum. Despite its widespread use for wide-sense stationary processes, the power spectrum is of limited utility for the present problem involving stationary increments, as we now show. Fix $L>0$ and consider the interval $[0, L]$. Almost every sample path $v(x, t)$ is bounded on $[0, L]$ and we may define the truncated Fourier transform

$$
\hat{v}_{L}(k, t)=\int_{0}^{L} e^{-i k x} v(x, t) d x .
$$

If the energy is finite $\left(M_{2}<\infty\right.$ in (72)), we may compute a truncated power spectral density $S_{L}(k)$ as follows. We have

$$
\frac{1}{L}\left|\hat{v}_{L}(k, t)\right|^{2}=\frac{1}{L} \int_{0}^{L} \int_{0}^{L} e^{-i k(x-y)} v(x, t) v(y, t) d x d y .
$$

Since $v(x, t)$ is a Lévy process with mean zero, the autocorrelation is

$$
\mathbb{E}(v(x, t) v(y, t))=(x \wedge y) M_{2} .
$$

We take expectations in (75) to find

$$
S_{L}(k):=\frac{1}{L} \mathbb{E}\left(\left|\hat{v}_{L}(k, t)\right|^{2}\right)=\frac{2 M_{2}}{k^{2}}\left(1-\frac{\sin k L}{k L}\right), \quad k \neq 0 .
$$

The power spectrum $S(k)=\lim _{L \rightarrow \infty} S_{L}(k)=2 M_{2} / k^{2}$ is now seen to be welldefined, but is unsuitable for distinguishing solutions because all solutions with the same energy (possibly infinite) have identical power spectrum.

A well-defined spectrum that distinguishes solutions may be obtained by taking the Fourier-Laplace transform of process paths. For fixed $p>0$ we define the random variable

$$
\mathcal{L} v(p, t)=\int_{0}^{\infty} e^{-p x} v(x, t) d x=\frac{1}{p} \int_{0}^{\infty} e^{-p x} v(d x, t) .
$$

The integrals are well-defined because $\lim _{x \rightarrow \infty} v(x, t) / x=0$ a.s. by the strong law of large numbers, and $v(x, t)$ is of bounded variation. If $s^{t}$ denotes a point process of shock strengths as in (41) we have

$$
p \mathcal{L} v(p, t)=\frac{M(t)}{p}-\sum_{0 \leq x} e^{-p x} s_{x}^{t} .
$$


We determine the law of $p \mathcal{L} v(p, t)$ by computing its Laplace transform via the 'infinitesimal' Lévy-Khintchine formula $\mathbb{E}\left(e^{q v(d x, t)}\right)=e^{\psi(q, t) d x}$. We then have

$$
\begin{aligned}
& \mathbb{E}\left(e^{q p \mathcal{L} v(p, t)}\right)=\exp \left(\int_{0}^{\infty} \psi\left(q e^{-p x}, t\right) d x\right) \\
& \quad=\exp \left(\frac{1}{p} \int_{0}^{q} \frac{\psi\left(q^{\prime}, t\right)}{q^{\prime}} d q^{\prime}\right)=: \exp \left(\frac{1}{p} \psi_{\#}(q, t)\right), \quad p, q>0,
\end{aligned}
$$

after the change of variables $q^{\prime}=q e^{-p x}$. We now observe that $\psi_{\#}$ determines a Laplace exponent as follows. Let $\bar{\Lambda}_{t}(s)=\int_{s}^{\infty} \Lambda_{t}(d s)$ denote the tail of the Lévy measure $\Lambda_{t}$. Since $\int_{0}^{\infty}\left(s \wedge s^{2}\right) \Lambda_{t}(d s)<\infty$ we have the bounds

$$
s \bar{\Lambda}_{t}(s) \leq \int_{s}^{\infty} r \Lambda_{t}(d r), \quad s^{2} \bar{\Lambda}_{t}(s) \leq \int_{0}^{\varepsilon} r^{2} \Lambda_{t}(d r)+s^{2} \bar{\Lambda}_{t}(\varepsilon), \quad s \in(0, \varepsilon) .
$$

Therefore,

$$
\lim _{s \rightarrow \infty} s \bar{\Lambda}_{t}(s)=0, \quad \lim _{s \rightarrow 0} s^{2} \bar{\Lambda}_{t}(s)=0,
$$

and we may integrate by parts in (30) to obtain

$$
\frac{\psi\left(q^{\prime}, t\right)}{q^{\prime}}=\int_{0}^{\infty}\left(1-e^{-q^{\prime} s}\right) \bar{\Lambda}_{t}(s) d s .
$$

Integrating once more in $q^{\prime}$ we find

$$
\psi_{\#}(q, t)=\int_{0}^{q} \frac{\psi\left(q^{\prime}, t\right)}{q^{\prime}} d q^{\prime}=\int_{0}^{\infty}\left(e^{-q s}-1+q s\right) \frac{\bar{\Lambda}_{t}(s)}{s} d s .
$$

We integrate by parts in (38) to see that

$$
\int_{0}^{\infty} \bar{\Lambda}_{t}(s) d s=\int_{0}^{\infty} s \Lambda_{t}(d s)=M(t)<\infty .
$$

This enables us to write

$$
\psi_{\#}(q, t)=M(t) q-\Phi_{\#}(q, t), \quad \Phi_{\#}(q, t)=\int_{0}^{\infty}\left(1-e^{-q s}\right) \frac{\bar{\Lambda}_{t}(s)}{s} d s .
$$

Since (83) ensures $s^{-1} \bar{\Lambda}_{t}(s) d s$ satisfies the finiteness conditions for a jump measure, $\psi_{\#}$ is a Laplace exponent for a Lévy process with zero mean drift that we denote by $Z^{t}$. Similarly, $\Phi_{\#}$ is the Laplace exponent for a subordinator that we denote $Y^{t}$. We summarize our calculations in the identities

$Z_{r}^{t}=M(t) r-Y_{r}^{t}, \mathbb{E}\left(e^{q Z_{r}^{t}}\right)=e^{r \psi_{\#}(q, t)}, \mathbb{E}\left(e^{-q Y_{r}^{t}}\right)=e^{-r \Phi_{\#}(q, t)}, r, q, t>0$. 
The result is that the Laplace spectrum of the solution increments is determined by

$$
\mathbb{E}\left(e^{q p \mathcal{L} v(p, t)}\right)=\mathbb{E}\left(e^{q Z_{1 / p}^{t}}\right), \quad q>0, p>0,
$$

which implies that $\mathcal{L} v(p, t)$ has the same law as $p^{-1} Z_{1 / p}^{t}$ for fixed $p>0$. Note that $\mathcal{L} v(p, t)$ is not a Lévy process in $p$. In fact, for a fixed realization, $\mathcal{L} v(p, t)$ is analytic in $p$. Nevertheless, its law is determined by the Lévy process $Z^{t}$.

We extend this computation to the Fourier spectrum $(p=i k)$ as follows. The calculations leading to (80) hold for complex $q$ with $\operatorname{Re}(q) \geq 0$, and in particular for $q=i \xi, \xi \in \mathbb{R}$. Moreover, $\mathcal{L} v(p, t)$ is a well-defined random variable for every $p$ with $\operatorname{Re}(p)>0$. Thus, we may analytically continue the identity $E\left(e^{q p \mathcal{L} v(p, t)}\right)=\exp \left(p^{-1} \psi_{\#}(q, t)\right)$ to all $p$ with $\operatorname{Re}(p)>0$, and $q=$ $i \xi$. As in (4), let $\Psi_{\#}(\xi, t)=-\psi_{\#}(i \xi, t)$ define the characteristic exponent corresponding to the Lévy process $Z^{t}$. For $\varepsilon, k>0$ we set $p=\varepsilon+i k$, $\hat{v}(k-i \varepsilon, t)=\mathcal{L} v(\varepsilon+i k, t)$ and pass to the limit $\varepsilon \rightarrow 0$ on both sides of (80) to obtain

$$
\begin{aligned}
\lim _{\varepsilon \downarrow 0} \mathbb{E}\left(e^{i \xi(i k \hat{v}(k-i \varepsilon, t))}\right)=\exp \left(\frac{1}{i k} \psi_{\#}(i \xi, t)\right) \\
\quad=\exp \left(\frac{i}{k} \Psi_{\#}(\xi, t)\right)=\mathbb{E}\left(e^{i \xi Z_{1 / k}^{t}}\right), \quad \xi \in \mathbb{R}, k>0 .
\end{aligned}
$$

Thus, for fixed $k>0$, as $\varepsilon \downarrow 0$ the random variables $i k \hat{v}(k-i \varepsilon, t)$ converge in law to the (real) random variable $Z_{1 / k}^{t}$. We denote this limit by $i k \hat{v}(k, t)$. As before we do not assert that the processes $i k \hat{v}(k-i \varepsilon, t)$ converge in law to the process $Z_{1 / k}^{t}$, simply the convergence of random variables for fixed $k$. We summarize our conclusions as follows.

Theorem 4. Let $Y^{t}$ be a subordinator with Laplace exponent $\Phi_{\#}(q, t)$ from (84), and let $Z^{t}$ the Lévy process defined by (85). Then for every fixed $p>0$ and $k>0$ the random variables $p \mathcal{L} v(p, t)$ and $i k \hat{v}(k, t)$ have the same law as $Z_{1 / p}^{t}$ and $Z_{1 / k}^{t}$, respectively.

Due to this result and (85), we always have the upper bound $i k^{2} \hat{v}(k, t) \leq$ $M(t)$ a.s. This crude bound may be refined as $k \rightarrow \infty$ using information related to the sample path behavior of subordinators (see [4, Ch. III.4]).

Corollary 1. For every $t>0, \lim _{k \rightarrow \infty} i k^{2} \hat{v}(k, t)=M(t)$ in probability. 
Proof. This follows from the fact that $\lim _{r \downarrow 0} Y_{r}^{t} / r=0$ in probability, proved as follows. By (85) we have $\mathbb{E}\left(e^{-q Y_{r}^{t} / r}\right)=e^{-r \Phi_{\#}(q / r, t)}$, and since $1-e^{-s} \leq$ $1 \wedge s$, by (84) we have that the Laplace exponent

$$
r \Phi_{\#}(q / r, t)=r \int_{0}^{\infty}\left(1-e^{-q s / r}\right) \frac{\bar{\Lambda}_{t}(s)}{s} d s \leq \int_{0}^{\infty}(r \wedge q s) \frac{\bar{\Lambda}_{t}(s)}{s} d s \rightarrow 0
$$

as $r \downarrow 0$ for each $q>0$. Hence $Y_{r}^{t} / r \rightarrow 0$ in law.

A similar conclusion holds for the Laplace spectrum as $p \rightarrow \infty$. Actually, for the subordinator $Y_{r}^{t}$, the sample paths have the stronger property that $\lim _{r \downarrow 0} Y_{r}^{t} / r \rightarrow 0$ a.s. [4, III.4.8]. Under a mild assumption on the integrability of the small jumps, we can strengthen convergence in probability to almost-sure convergence of the Laplace spectrum.

Corollary 2. For every $t>0, \lim _{p \rightarrow \infty} p^{2} \mathcal{L} v(p, t)=M(t)$ in probability. If we also assume $\int_{0}^{1}|\log s| \bar{\Lambda}_{t}(s) d s<\infty$, then $\lim _{p \rightarrow \infty} p^{2} \mathcal{L} v(p, t)=M(t)$ a.s.

Proof. For notational convenience, we suppress the dependence on $t$ in the proof. Fix $\varepsilon>0$, and let $p_{m}=2^{m}$ for positive integers $m$. We will show that $\lim _{m \rightarrow \infty} p_{m}^{2} \mathcal{L} v\left(p_{m}\right)=M$ a.s. That is, for every $\varepsilon>0$, we claim

$$
\mathbb{P}\left(\left|p_{m}^{2} \mathcal{L} v\left(p_{m}\right)-M\right|>\varepsilon \text { infinitely often }\right)=0 .
$$

This is sufficient to establish $\lim _{p \rightarrow \infty} p^{2} \mathcal{L} v(p)=M$ a.s. Indeed, since $M / p-$ $p \mathcal{L} v(p)$ is completely monotone by (79), for $p \in\left(p_{m}, p_{m+1}\right)$ we have the bounds

$$
0<M(t)-p^{2} \mathcal{L} v(p)<\frac{p}{p_{m}}\left(M(t)-p_{m}^{2} \mathcal{L} v\left(p_{m}\right)\right)<2\left(M-p_{m}^{2} \mathcal{L} v\left(p_{m}\right)\right),
$$

and therefore

$$
\left\{M-p^{2} \mathcal{L} v(p)>2 \varepsilon\right\} \subset\left\{M-p_{m}^{2} \mathcal{L} v\left(p_{m}\right)>\varepsilon\right\} .
$$

In order to prove (89) we use the elementary estimate

$$
\begin{aligned}
& \mathbb{P}\left(\left|p_{m}^{2} \mathcal{L} v(p)-M\right|>\varepsilon\right)=\mathbb{P}\left(p_{m} Y_{1 / p_{m}}>\varepsilon\right) \leq \frac{e}{e-1} \mathbb{E}\left(1-\exp \left(-\frac{p_{m}}{\varepsilon} Y_{1 / p_{m}}\right)\right) \\
& =\frac{e}{e-1}\left(1-\exp \left(-\frac{1}{p_{m}} \Phi_{\#}\left(\frac{p_{m}}{\varepsilon}\right)\right)\right) \leq \frac{e}{e-1} \frac{1}{p_{m}} \Phi_{\#}\left(\frac{p_{m}}{\varepsilon}\right) .
\end{aligned}
$$

We will show that $\sum_{m=1}^{\infty} p_{m}^{-1} \Phi_{\#}\left(p_{m} / \varepsilon\right)<\infty$. The first Borel-Cantelli lemma then implies (89). For clarity, we suppose $\varepsilon=1$. This causes no essential difference and reveals the main computation. 
Denote the integrated tail of the Lévy measure for $Y^{t}$ by

$$
\Pi_{t}(s)=\int_{s}^{\infty} \frac{\bar{\Lambda}_{t}\left(s^{\prime}\right)}{s^{\prime}} d s^{\prime}
$$

We integrate by parts and use Tonelli's theorem to find

$$
\sum_{m=1}^{\infty} p_{m}^{-1} \Phi_{\#}\left(p_{m}\right)=\int_{0}^{\infty} \sum_{m=1}^{\infty} e^{-p_{m} s} \Pi_{t}(s) d s .
$$

It is only necessary to check that the integral over $s \in(0,1)$ is finite. Here we use the elementary estimate

$$
\sum_{m=1}^{\infty} e^{-2^{m} s} \leq \int_{0}^{\infty} \exp \left(-e^{x \log 2} s\right) d x=\frac{1}{\log 2} \int_{s}^{\infty} e^{-y} \frac{d y}{y} \leq \frac{|\log s|+1}{\log 2}
$$

so that

$$
\int_{0}^{1} \sum_{m=1}^{\infty} e^{-p_{m} s} \Pi_{t}(s) d s \leq \frac{1}{\log 2} \int_{0}^{1}(1+|\log s|) \Pi_{t}(s) d s .
$$

By the definition of $\Pi_{t}(s)$ in (91), the last integral is

$$
\begin{gathered}
\int_{0}^{1}|\log s| \int_{s}^{\infty} \frac{\bar{\Lambda}_{t}(r)}{r} d r d s=\int_{0}^{\infty} \frac{\bar{\Lambda}_{t}(r)}{r} d r \int_{0}^{1 \wedge r}|\log s| d s \\
\leq \int_{0}^{1}|\log r| \bar{\Lambda}_{t}(r) d r+\int_{0}^{\infty} \bar{\Lambda}_{t}(r) d r,
\end{gathered}
$$

which is finite by assumption.

Corrections to the bound $i k^{2} \hat{v}(k, t) \leq M(t)$ involve the law of the iterated logarithm [4, III.4]. The following corollary holds for initial data that is not BV (so $M_{0}=+\infty$ and $M(t)=1 / t$ ) with suitably regular small jumps ('dust').

Corollary 3. Assume $\sigma_{0} \neq 0$ and $\alpha=2$, or assume $\sigma_{0}=0$ and $\bar{\Lambda}_{0}(s)=$ $\int_{s}^{\infty} \Lambda(d r)$ is regularly varying at zero with exponent $-\alpha$ where $\alpha \in(1,2)$. Then for every $c>0$ and $t>0$ we have

$$
-\log \mathbb{P}\left(\frac{t^{-1}-i k^{2} \hat{v}(k, t)}{h(k \log \log k)} \leq c\right) \sim \frac{\log \log k}{\gamma c^{\gamma} t^{1+2 \gamma}}, \quad k \rightarrow \infty,
$$

where $\gamma=1 /(\alpha-1)$ and $h(k)=k / \psi_{0}(k)$. 
This corollary is a consequence of [4, Lemma III.12] and is associated with the following lemma of independent interest which shows that the evolution preserves the regularity of the dust.

Lemma 2. (a) Assume that $\sigma_{0}=0$ and $\bar{\Lambda}_{0}(s)$ is regularly varying at zero with exponent $-\alpha, \alpha \in(1,2)$. Then $\bar{\Lambda}_{t}(s)$ is regularly varying at zero with exponent $-1 / \alpha$ for every $t>0$.

(b) If $\sigma_{0} \neq 0$, then $\bar{\Lambda}_{t}(s) \sim\left(\sigma_{0} t\right)^{-1} \sqrt{2 /(\pi s)}$ as $s \rightarrow 0$, for every $t>0$.

Proof. Recall that $t^{-1}-i k^{2} \hat{v}(k, t)$ agrees in law with $k Y_{1 / k}^{t}$. Combining (28) with (82) we find that the Laplace exponent of the subordinator $Y^{t}$ satisfies

$$
\Phi_{\#}(q, t)=\int_{0}^{q} \Phi\left(\frac{q^{\prime}}{t}, t\right) \frac{d q^{\prime}}{q^{\prime}}=\int_{0}^{q / t} \Phi\left(q^{\prime}, t\right) \frac{d q^{\prime}}{q^{\prime}} .
$$

We claim that $\Phi(\cdot, t)$, and hence $\Phi_{\#}(\cdot, t)$, is regularly varying at $\infty$ with exponent $\hat{\alpha}=1 / \alpha \in\left[\frac{1}{2}, 1\right)$.

To prove the claim, we integrate by parts in (25) to obtain

$$
\frac{\psi_{0}(q)}{q^{2}}=\frac{\sigma_{0}^{2}}{2}+\int_{0}^{\infty} e^{-q s}\left(\int_{s}^{\infty} \bar{\Lambda}_{0}(r) d r\right) d s
$$

First assume $\sigma_{0}=0$ and $\bar{\Lambda}_{0}$ is regularly varying at zero with exponent $-\alpha$. Then $\psi_{0}$ is regularly varying at infinity with exponent $\alpha$. This follows from [18, XIII.5.3], or may be proved directly. If $\sigma_{0} \neq 0$, we have $\lim _{q \rightarrow \infty} \psi_{0}(q) / q^{2}=\sigma_{0}^{2} / 2$. The Laplace exponent $\Phi(q, t)$ is determined via the functional relation (32). Since $\alpha>1$, the map $\Phi \mapsto g_{0}(\Phi):=\psi_{0}(t \Phi)+\Phi$ is regularly varying (in $\Phi$ ) at $\infty$ with exponent $\alpha$. Therefore, the inverse function $\Phi(q, t)$ is regularly varying (in $q$ ) at $\infty$ with exponent $1 / \alpha$.

Now let $g_{\#}(\cdot, t)$ be the inverse function to $\Phi_{\#}(\cdot, t)$. Then by $[4$, Lemma III.4.12] we infer that for every $\hat{c}>0$,

$$
-\log \mathbb{P}\left(\frac{k Y_{1 / k}^{t}}{h_{\#}(k \log \log k, t)} \leq \hat{c}\right) \sim(1-\hat{\alpha})(\hat{\alpha} / \hat{c})^{\hat{\alpha} /(1-\hat{\alpha})} \log \log k, \quad k \rightarrow \infty,
$$

where

$$
h_{\#}(k, t)=\frac{k}{g_{\#}(k, t)} .
$$

By (94) and regular variation we have $\Phi_{\#}(q, t) \sim \Phi(q / t, t) / \hat{\alpha}$ as $q \rightarrow \infty$, and thus by (32) we find that as $q \rightarrow \infty$,

$$
g_{\#}(q, t) \sim t g_{0}(\hat{\alpha} q) \sim t \psi_{0}(\hat{\alpha} t q) \sim t^{1+\alpha} \alpha^{-\alpha} \psi_{0}(q) .
$$


Substituting $\hat{c}=c t^{1+\alpha} \alpha^{-\alpha}$ into (95) yields Corollary 3 .

Karamata's Tauberian theorem and the monotone density theorem now imply that $\bar{\Lambda}_{t}(s)$ is regularly varying at zero with exponent $-1 / \alpha$. If $\sigma_{0} \neq 0$, we find $\Phi(q, t) \sim\left(\sigma_{0} t\right)^{-1} \sqrt{2 q}$ as $q \rightarrow \infty$. Assertion (b) of the Lemma then follows from the Tauberian theorem.

For the self-similar solutions, $\psi_{0}(q)=q^{\alpha}$ with $\alpha \in(1,2], \bar{\Lambda}_{0}(s)=$ $s^{-\alpha} /(\alpha \Gamma(-\alpha))$ for $\alpha \in(1,2)$, and we have $\bar{\Lambda}_{t}(s) \sim t^{-1}(t s)^{-1 / \alpha} / \Gamma(1-1 / \alpha)$ as $s \rightarrow 0$.

\subsection{The multifractal spectrum}

The notion of a multifractal spectrum was introduced by Frisch and Parisi to describe the intermittency of velocity fields in fully developed turbulence [20]. The multifractal spectrum $d(h)$ measures the dimension of the set $S_{h}$ where the velocity field has singularities of order $h$. There are different mathematical formulations of multifractality, corresponding to different notions of what one means by singularities of order $h$. Here we follow the treatment by Jaffard, which yields $d(h)$ rather easily [29] (the notation has been changed slightly for consistency with this article).

We say a function $v: \mathbb{R}_{+} \rightarrow \mathbb{R}$, is $C^{r}\left(x_{0}\right)$ for a point $x_{0} \in \mathbb{R}_{+}$if there is a polynomial $P_{x_{0}}$ of degree at most $[r]$ such that

$$
\left|v(x)-P_{x_{0}}(x)\right| \leq C\left|x-x_{0}\right|^{r},
$$

in a neighborhood of $x_{0}$. The Hölder exponent of $v$ at $x_{0}$ is defined as

$$
h_{v}\left(x_{0}\right)=\sup \left\{r \mid v \in C^{r}\left(x_{0}\right)\right\} .
$$

We define $S_{h}$ to be the set of points where $v$ is of Hölder exponent $h$. The multifractal spectrum $d(h)$ is the Hausdorff dimension of $S_{h}$. If $S_{h}$ is empty, the convention is $d(h)=-\infty$. As an example, let us compute the multifractal spectrum when the initial data is of bounded variation. Then $M_{0}<\infty$ in (41) and there is a finite number of shocks $s_{y}^{t}$ in a finite interval $[0, x]$ with probability 1 . Suppose $x_{0}$ is not a shock location for $v(\cdot, t)$. Then (41) shows $v$ is analytic near $x_{0}$ and $h_{v}\left(x_{0}\right)=\infty$. If $x_{0}$ is a shock location, then $v \in C^{-\varepsilon}\left(x_{0}\right)$ for every $\varepsilon>0$, so that $h_{v}\left(x_{0}\right)=0$. Thus, we have simply $d(0)=0$ and $d(h)=-\infty$ for every $h \neq 0$.

The multifractal spectrum is more interesting for initial data of unbounded variation, that is, when (39) holds. In this case, the jumps in 
$v$ are dense. Following Jaffard [29], the multifractal spectrum is computed as follows. We define

$$
C_{j}(t)=\int_{2^{-j-1}}^{2^{-j}} \Lambda_{t}(d s), \quad \tilde{\beta}_{t}=\max \left(0, \limsup _{j \rightarrow \infty} \frac{\log C_{j}(t)}{j \log 2}\right) .
$$

For any $t>0, v(\cdot, t)$ has no Brownian component. It then follows from [29, Thm. 1] that

$$
d_{t}(h)= \begin{cases}\tilde{\beta}_{t} h, & h \in\left[0,1 / \tilde{\beta}_{t}\right] \\ -\infty, & \text { else. }\end{cases}
$$

Experiments suggest that the multifractal spectrum for fully developed threedimensional turbulence is a concave curve [31, Fig. 2]. This is in clear contrast with (97).

For example, let us compute the multifractal spectrum for the self-similar process $V^{\alpha}$ of index $\alpha \in(1,2]$. Since $\Lambda_{t}^{\alpha}(s)$ is a scaled copy of $\Lambda_{1}^{\alpha}(s), d_{t}(h)$ is independent of $t$. We use (7) to obtain the asymptotics as $s \rightarrow 0$ :

$$
\Lambda_{1}^{\alpha}(d s)=f_{\alpha}(s) d s \sim \frac{\sin \pi \beta}{\pi} s^{\beta-2} \Gamma(2-\beta) d s, \quad \beta=\frac{\alpha-1}{\alpha} .
$$

We then have $\tilde{\beta}_{t}=\alpha^{-1}, t>0$ and

$$
d(h)= \begin{cases}h / \alpha, & h \in[0, \alpha] \\ -\infty, & \text { else. }\end{cases}
$$

In particular, (98) implies that $d(\alpha)=1$, that is $v(x, t)$ is $C^{\alpha}(x)$ for a.e $x \in \mathbb{R}_{+}$. For this set a finer characterization of the local variation of $v(\cdot, t)$ may be obtained by using the Fristedt-Pruitt law of the iterated logarithm (see [5, Cor. 1]). However, the multifractal spectrum, also describes sets $S_{h}$, $0<h<\alpha$, that are not covered by the Fristedt-Pruitt law.

\section{Acknowledgement}

This material is based upon work supported by the National Science Foundation under grants DMS 03-05985 and DMS 04-05343. G.M. thanks the University of Crete for hospitality during part of this work.

\section{References}

[1] M. Abramowitz And I. A. Stegun, Handbook of mathematical functions with formulas, graphs, and mathematical tables, vol. 55 of National 
Bureau of Standards Applied Mathematics Series, For sale by the Superintendent of Documents, U.S. Government Printing Office, Washington, D.C., 1964.

[2] D. J. Aldous, Deterministic and stochastic models for coalescence (aggregation and coagulation): a review of the mean-field theory for probabilists, Bernoulli, 5 (1999), pp. 3-48.

[3] M. Avallaneda and W. E, Statistical propeties of shocks in Burgers turbulence, Comm. Math. Phys., 172 (1995), pp. 13-38.

[4] J. Bertoin, Lévy processes, vol. 121 of Cambridge Tracts in Mathematics, Cambridge University Press, Cambridge, 1996.

[5] —- The inviscid Burgers equation with Brownian initial velocity, Comm. Math. Phys., 193 (1998), pp. 397-406.

[6] _- Clustering statistics for sticky particles with Brownian initial velocity, J. Math. Pures Appl. (9), 79 (2000), pp. 173-194.

[7] _- Eternal solutions to Smoluchowski's coagulation equation with additive kernel and their probabilistic interpretations, Ann. Appl. Probab., 12 (2002), pp. 547-564.

[8] — Some aspects of additive coalescents, in Proceedings of the International Congress of Mathematicians, Beijing 2002, vol. III, Higher Ed. Press, 2002, pp. 15-23.

[9] N. H. Bingham, Maxima of sums of random variables and suprema of stable processes, Z. Wahrscheinlichkeitstheorie und Verw. Gebiete, 26 (1973), pp. 273-296.

[10] J. M. Burgers, The nonlinear diffusion equation, Dordrecht: Reidel, 1974.

[11] L. Carraro and J. Duchon, Solutions statistiques intrinsèques de l'équation de Burgers et processus de Lévy, C. R. Acad. Sci. Paris Sér. I Math., 319 (1994), pp. 855-858.

[12] —_Équation de Burgers avec conditions initiales à accroissements indépendants et homogènes, Ann. Inst. H. Poincaré Anal. Non Linéaire, 15 (1998), pp. 431-458.

[13] M.-L. Chabanol And J. Duchon, Markovian solutions of inviscid Burgers equation, J. Statist. Phys., 114 (2004), pp. 525-534. 
[14] P. Chassaing And G. Louchard, Phase transition for parking blocks, Brownian excursion and coalescence,, Random Structures and Algorithms, 21 (2002), pp. 76-119.

[15] J. D. Cole, On a quasi-linear parabolic equation occurring in aerodynamics, Quart. Appl. Math., 9 (1951), pp. 225-236.

[16] R. L. Drake, A general mathematical survey of the coagulation equation, in Topics in Current Aerosol Research, G. M. Hidy and J. R. Brock, eds., no. 2 in International reviews in Aerosol Physics and Chemistry, Pergammon, 1972, pp. 201-376.

[17] W. E AND Y. G. SinA $\breve{I}$, New results in mathematical and statistical hydrodynamics, Uspekhi Mat. Nauk, 55 (2000), pp. 25-58.

[18] W. FELLER, An introduction to probability theory and its applications. Vol. II., Second edition, John Wiley \& Sons Inc., New York, 1971.

[19] L. Frachebourg and P. A. Martin, Exact statistical properties of the Burgers equation, J. Fluid Mech., 417 (2000), pp. 323-349.

[20] U. Frisch AND G. PARISI, On the singularity structure of fully developed turbulence, in Turbulence and predictability in geophysics, M. Ghil, R. Benzi, and R.Parisi, eds., North-Holland, 1985, pp. 8487.

[21] T. Funaki, D. Surgailis, and W. A. Woyczyński, Gibbs-Cox random fields and Burgers turbulence, Ann. Appl. Probab., 5 (1995), pp. 461-492.

[22] C. Giraud, Genealogy of shocks in Burgers turbulence with white noise initial velocity, Comm. Math. Phys., 223 (2001), pp. 67-86.

[23] A. M. Golovin, The solution of the coagulating equation for cloud droplets in a rising air current, Izv. Geophys. Ser., (1963), pp. 482487.

[24] P. Groeneboom, Brownian motion with a parabolic drift and Airy functions, Probab. Theory Related Fields, 81 (1989), pp. 79-109.

[25] S. N. Gurbatov, A. N. Malakhov, and A. I. Saichev, Nonlinear random waves and turbulence in nondispersive media: waves, rays, particles, Manchester University Press, Manchester, 1991. 
[26] S. N. Gurbatov, S. I. Simdyankin, E. Aurell, U. Frisch, and G. Tóth, On the decay of Burgers turbulence, J. Fluid Mech., 344 (1997), pp. 339-374.

[27] E. Hopf, The partial differential equation $u_{t}+u u_{x}=\mu_{x x}$, Comm. Pure Appl. Math., 3 (1950), pp. 201-230.

[28] J. JaCod And A. N. ShiRyaev, Limit theorems for stochastic processes, vol. 288 of Grundlehren der Mathematischen Wissenschaften, Springer-Verlag, Berlin, second ed., 2003.

[29] S. Jaffard, The multifractal nature of Lévy processes, Probab. Theory Related Fields, 114 (1999), pp. 207-227.

[30] A. N. Kolmogorov, Dissipation of energy in the locally isotropic turbulence, Proc. Roy. Soc. London Ser. A, 434 (1991), pp. 15-17. Translated from the Russian by V. Levin, Turbulence and stochastic processes: Kolmogorov's ideas 50 years on.

[31] C. Meneveau and K. R. Sreenivasan, Simple multifractal cascade model for fully developed turbulence, Phys. Rev. Lett., 59 (1987), pp. 1424-1427.

[32] G. Menon And R. Pego, Approach to self-similarity in Smoluchowski's coagulation equations, Comm. Pure Appl. Math., 57 (2004), pp. 1197-1232.

[33] S. RESNICK, Extreme values, regular variation and point processes, Springer-Verlag, New York, 1987.

[34] Z.-S. She, E. Aurell, AND U. Frisch, The inviscid Burgers equation with initial data of Brownian type, Comm. Math. Phys., 148 (1992), pp. 623-641.

[35] Y. G. Sina I, Statistics of shocks in solutions of inviscid Burgers equation, Comm. Math. Phys., 148 (1992), pp. 601-621.

[36] W. A. Woyczyński, Burgers-KPZ turbulence, vol. 1700 of Lecture Notes in Mathematics, Springer-Verlag, Berlin, 1998. Göttingen lectures.

[37] R. M. ZIFF, Kinetics of polymerization, J. Statist. Phys., 23 (1980), pp. 241-263. 